\title{
A Twin Spiral Planar Antenna for UWB Medical Radars
}

\author{
Giuseppe A. Zito, ${ }^{1}$ Enrico M. Staderini, ${ }^{2}$ and Stefano Pisa ${ }^{3}$ \\ ${ }^{1}$ Gerontechnology and Rehabilitation Group, ARTORG Center for Biomedical Engineering Research, University of Bern, \\ 3010 Bern, Switzerland \\ ${ }^{2}$ Haute École d'Ingénierie et de Gestion du Canton de Vaud, Western Switzerland University of Applied Sciences (HES-SO), \\ 1401 Yverdon-les-Bains, Switzerland \\ ${ }^{3}$ Department of Information Engineering, Electronics and Telecommunications, Sapienza University of Rome, 00184 Rome, Italy
}

Correspondence should be addressed to Stefano Pisa; pisa@die.uniroma1.it

Received 1 October 2012; Accepted 19 November 2012

Academic Editor: Renato Cicchetti

Copyright (C) 2013 Giuseppe A. Zito et al. This is an open access article distributed under the Creative Commons Attribution License, which permits unrestricted use, distribution, and reproduction in any medium, provided the original work is properly cited.

A planar-spiral antenna to be used in an ultrawideband (UWB) radar system for heart activity monitoring is presented. The antenna, named "twin," is constituted by two spiral dipoles in a compact structure. The reflection coefficient at the feed point of the dipoles is lower than $-8 \mathrm{~dB}$ over the $3-12 \mathrm{GHz}$ band, while the two-dipoles coupling is about $-20 \mathrm{~dB}$. The radiated beam is perpendicular to the plane of the spiral, so the antenna is wearable and it may be an optimal radiator for a medical UWB radar for heart rate detection. The designed antenna has been also used to check some hypotheses about the UWB radar heart activity detection mechanism. The radiation impedance variation, caused by the thorax vibrations associated with heart activity, seems to be the most likely explanation of the UWB radar operation.

\section{Introduction}

Ultrawideband radar systems transmit and receive ultrashort pulses having ultrawide bandwidth and very low power levels [1-5]. These properties make UWB radars safe for the exposed human being and compliant with other apparatuses in the environment, but at the same time increase the difficulty for the echo signal detection. For these reasons the radar antenna plays a crucial role in the UWB systems; in fact, it should combine the flat frequency response requirement on a wide band with directivity properties. Generally, a radiated beam directed towards the body is preferred for body-worn devices in order to minimize the "losses" towards the environment.

Some kinds of UWB antennas, as horn or Vivaldi antennas, have an high directivity [6-9]. However, for all these radiators the maximum directivity is in the same direction of the maximum size of the antenna. In order to realize a wearable radar system, a directivity in a direction perpendicular to the maximum size of the antenna is necessary. For this reason, other kinds of devices have to be investigated as, for example, spiral antennas. These antennas are constituted by two coplanar spirals that unwind with a given flare rate [10]. The main characteristic of spiral antennas is a radiated beam, very constant with the frequency, pointing in two opposite directions perpendicular to the spiral plan. An implementation of this antenna uses a perfect electric conducting (PEC) ground plane placed at a certain distance below the antenna to produce a unidirectional beam [11]. Finally, it is important to note that all the cited antennas can be used in a UWB radar to observe copolarized reflections. For the heart activity monitoring the use of cross-polarized antennas could be advantageous. In fact, with respect to copolarized radiators, cross-polarized antennas reduce the backscattering from planar surfaces, as the chest wall, and increase the backscattered field from asymmetric structures like the heart $[12,13]$.

Another open issue in UWB radar technique for heart activity monitoring is the physical rationale at the basis of the measured signals. After the first hypothesis, based on the far field operation, reported in the McEwan's patent [14], a wide set of hypotheses has been tested in [15]. Among others, 


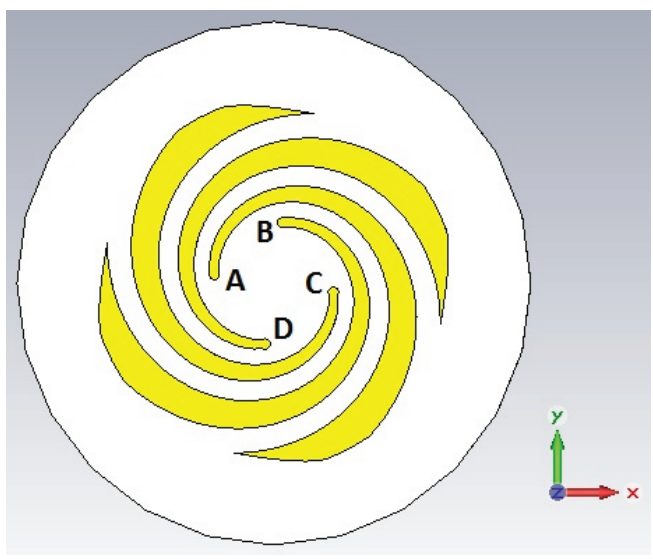

(a)

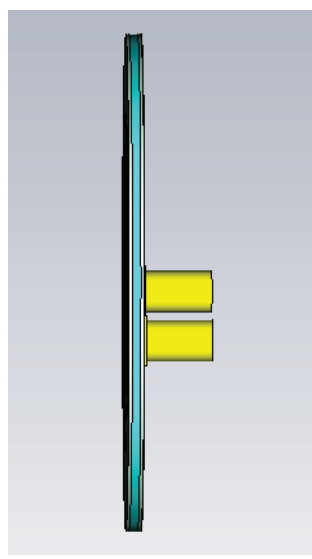

(b)

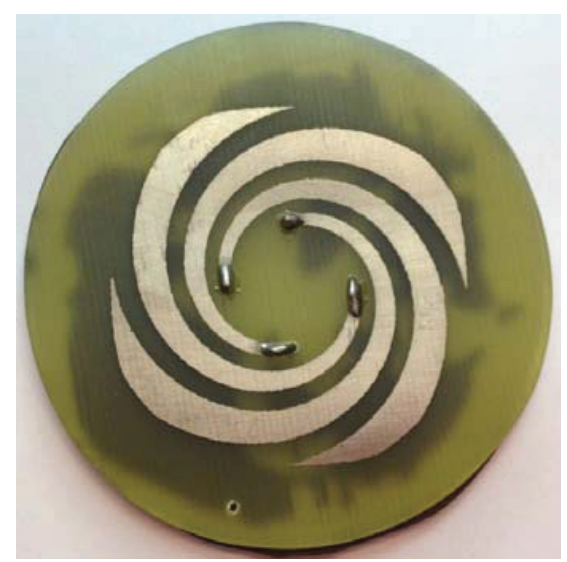

(c)

FIGURE 1: Geometry of the proposed twin spiral antenna. Frontal view (a) and lateral view (b). Antenna physical realization (c).

in [15] the blood perfusion in the skin layer and in all the thorax layers and the micromovements of the skin due to the heartbeat have been considered. However no definitive solution has been given to this problem.

In this paper a novel small-size directional twin crosspolarized planar spiral antenna for UWB systems is proposed. It is based on two twin spiral antennas rotated by $180^{\circ}$ to each other on the same plane, one is used in transmission and the other in reception. Moreover, by using the designed antenna and a multilayer model of the human body, a theoretical study is performed for investigating the interaction mechanisms at the basis of the UWB radar operation.

\section{Antenna Design}

The main goal of the antenna design is to obtain, in the $3.1-10.6 \mathrm{GHz}$ frequency range, issued by the federal communication commission (FCC) guidelines for medical applications [16], a reflection coefficient $S_{11}$ and a coupling coefficient $S_{21}$ lower than $-8 \mathrm{~dB}$ and $-20 \mathrm{~dB}$, respectively.

The chosen antenna geometry is reported in Figure 1(a). The general equation for the upper and lower parts of the spiral arms on the $x-y$ plane are

$$
\begin{aligned}
& x(t)=a^{t / b} \cos (t) \\
& y(t)=a^{t / b} \sin (t),
\end{aligned}
$$

where " $a$ " and " $b$ " are parameters to be optimized to satisfy the design goals while " $t$ " is the running variable. The final part of the lower line is closed with the higher part using a 4 point Spline. The other three arms of the antenna are obtained rotating the first arm of $90^{\circ}, 180^{\circ}$, and $270^{\circ}$ around $z$-axis.

The twin spiral geometry has been printed on a substrate having circular geometry, with a radius equal to $30 \mathrm{~mm}$. The used substrate is a sheet of FR-4 having a relative permittivity $\varepsilon_{r}=4.3$ and thickness $h=0.4 \mathrm{~mm}$. In order to achieve an antenna pattern mainly in the direction of positive $z$-axis, an absorbing material and a reflecting plate have been placed behind the antenna. The absorbing material is ECCOSORBFGM-40 with $1 \mathrm{~mm}$ thickness, the reflecting element is the copper plate of an FR-4 substrate. SMA connectors have been placed between point A in Figure 1 and the ground (port 1) and between point $\mathrm{D}$ and the ground (port 2) (see Figure $1(b)$ ) while points $B$ and $C$ have been short circuited to the ground. In this manner a fully unbalanced structure is realized allowing an easy integration of this antenna in UWB radars with unbalanced TX/RX connections [17].

The antenna geometry has been optimized through parametric simulations using CST Microwave Studio software. In these simulations the antenna parameters ( $a$ and $b$ in (1)) have been varied inside realistic ranges and magnitudes of the $S_{11}(f)$ and $S_{21}(f)$ scattering coefficients have been analyzed. Only geometries giving $S_{11}(f)$ magnitude lower than $-8 \mathrm{~dB}$ $\left(\rho_{11}=0.4\right)$ and $S_{21}(f)$ magnitude lower than $-20 \mathrm{~dB}\left(\rho_{21}=\right.$ 0.1 ) between $f_{1}=3.1 \mathrm{GHz}$ and $f_{2}=10.6 \mathrm{GHz}$ have been considered and, among those selected, the one maximizing the cost function

$$
C=\frac{1}{\left(f_{2}-f_{1}\right)} \int_{f_{1}}^{f_{2}}\left(\frac{\rho_{11}-\left|S_{11}(f)\right|}{\rho_{11}}\right) \cdot\left(\frac{\rho_{21}-\left|S_{21}(f)\right|}{\rho_{21}}\right) d f
$$

has been chosen. The best value of (2) has been obtained with " $a$ " equal to 2.55 and 2.75 for the lower and the upper lines of the arm, respectively, and " $b$ " equal to 4 .

\section{Numerical Simulations and Measured Results}

Figure 2 shows the return loss $S_{11}$ (a) and the isolation $S_{21}$ (b) as a function of the frequency obtained by means of CST simulations of the optimized antenna. The simulated results indicate that, according to the design goals, the antenna has a return loss lower than $-8 \mathrm{~dB}$ and the $S_{21}$ is always less than $-20 \mathrm{~dB}$ in the $3.1-10.6 \mathrm{GHz}$ frequency band.

The designed antenna has been realized (see Figure 1(c)) and the $S_{11}$ and $S_{21}$ scattering parameters have been measured by means of a vector network analyzer (PNA E8363B). The 


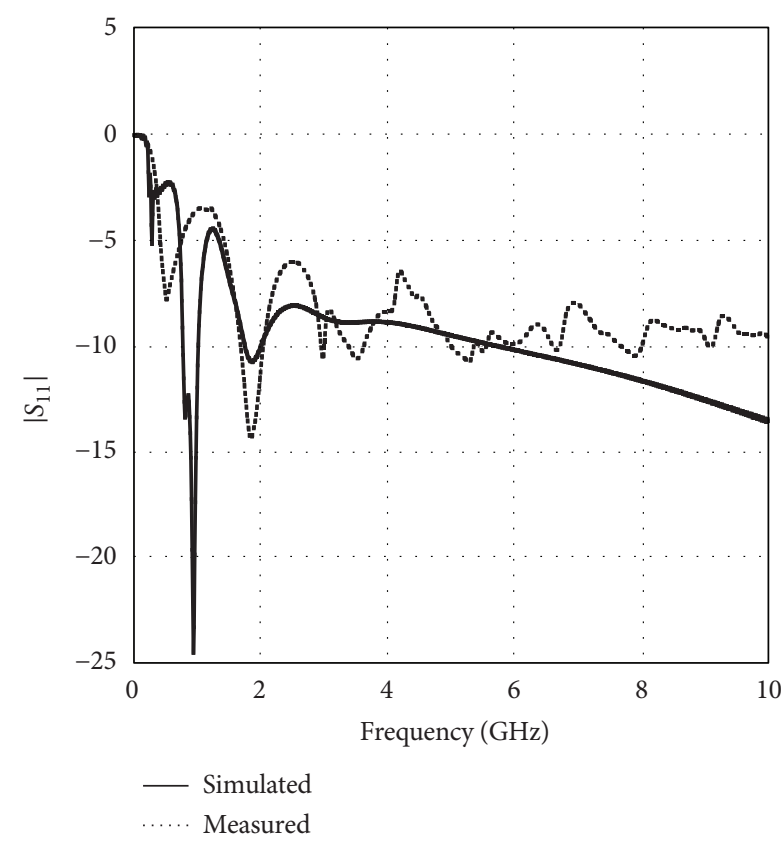

(a)

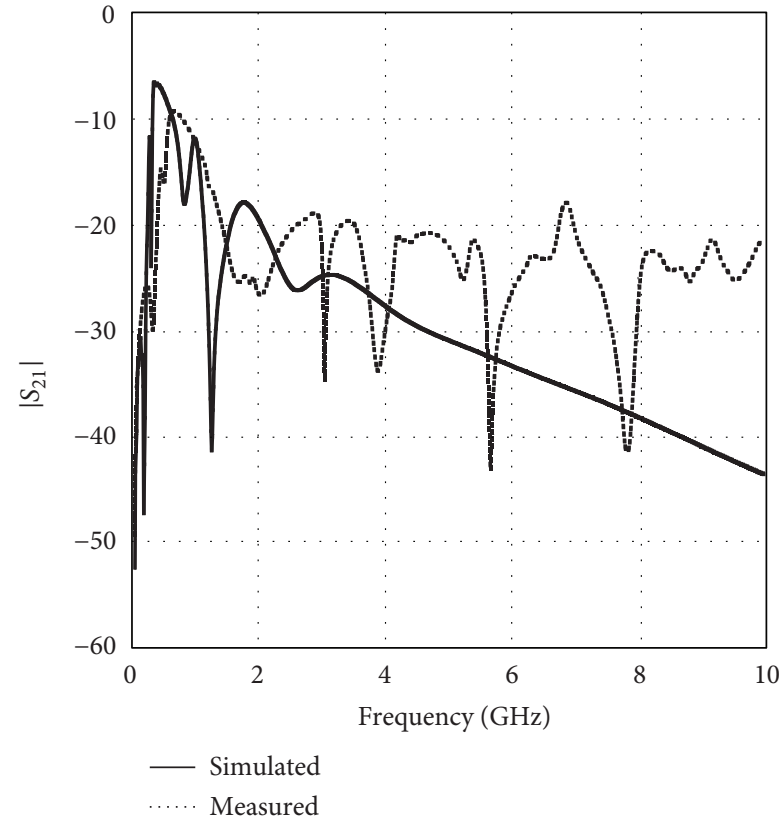

(b)

FIGURE 2: Return loss $S_{11}$ (a) and isolation $S_{21}$ (b) as a function of the frequency. Comparison between simulated and measured results.

obtained results are reported in Figures 2(a) and 2(b), respectively. The figures show that the $S_{11}$ frequency behaviors are in a quite good agreement with simulations, while some discrepancies, probably due to the SMA connectors, are present in the $S_{21}$ behavior.

Figure 3 shows the simulated antenna radiation pattern, on the $x-z$ plane (a) and on the $y-z$ plane (b) at $4,6,8$, and $10 \mathrm{GHz}$. The plots highlight that the maximum radiation is on the $z$ direction with a $-3 \mathrm{~dB}$ aperture of about $70^{\circ}$. Notice that in Figure 3(a) the main lobe direction is deviated with respect the $z$ axis of about $15^{\circ}$ : this behavior was not present in simulations without SMA connectors.

An important parameter for characterizing a UWB antenna is the fidelity factor that is the peak value of the crosscorrelation function between the signal $s_{2}(t)$ (electric field), at a given distance from the antenna, and the signal $s_{1}(t)$ (input voltage) [18]

$$
F=\max _{\tau} \frac{\int_{-\infty}^{\infty} s_{1}(t) s_{2}(t+\tau) d t}{\sqrt{\int_{-\infty}^{\infty} s_{1}^{2}(t) d t} \sqrt{\int_{-\infty}^{\infty} s_{2}^{2}(t) d t}},
$$

where $\tau$ is the delay that maximizes $F$ in (3).

The fidelity factor has been calculated in air at various distances from the antenna and for various directions. Results concerning the fidelity are summarized in Table 1 . The values reported in the table highlight the very high fidelity factor of the proposed antenna.

\section{Operating Principles}

The first hypothesis on the UWB radar operating principle was proposed by McEwans [14] and was based on the far field
TABLE 1: Simulated fidelity factor for the proposed twin antenna.

\begin{tabular}{lc}
\hline Probe position $(\mathrm{cm})$ & Fidelity factor \\
\hline$x=0 ; y=0 ; z=10$ & 0.9803 \\
$x=0 ; y=0 ; z=5$ & 0.9732 \\
$x=4 ; y=0 ; z=0$ & 0.9283 \\
$x=-4 ; y=0 ; z=0$ & 0.9680 \\
\hline
\end{tabular}

detection of heart wall movements. In [15] the authors have evidenced that a UWB radar cannot detect the movements of the cardiac wall because of the strong attenuation of the UWB pulse in the thorax. A possible operating mechanism suggested in [15] is based on a near field interaction: the detected signal is not an echo, but the consequence of a change in the antenna radiation impedance at the same frequency of the heart rate.

To investigate this phenomenon, two possible mechanisms have been hypothesized, in particular the increase of the blood perfusion in the skin layer and in all the thorax layers, and the micromovements of the skin due to the heartbeat [15].

In order to study the influence of the blood perfusion on the antenna radiation impedance, first of all the alteration in the tissue dielectric properties due to the blood increase caused by heart activity has been quantified. For this purpose, a human body and lung volume of 70 and 5 liters, respectively, have been considered assuming a stroke volume of $80 \mathrm{~mL}$ flowing both in the lung and in the other tissues according to the big and small circulation concepts. Moreover, the whole blood content has been assumed equal to 5 liters uniformly distributed in the body. Notice that this is a rough 


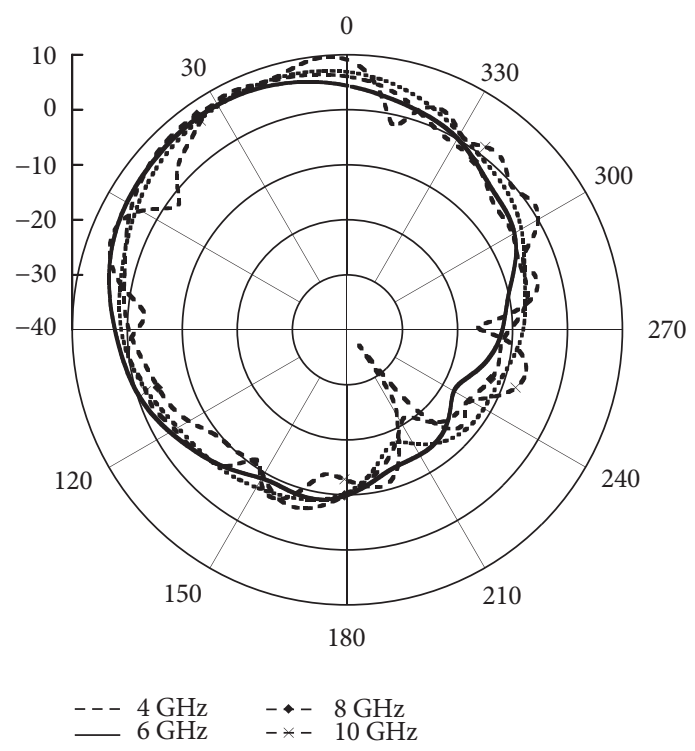

(a)

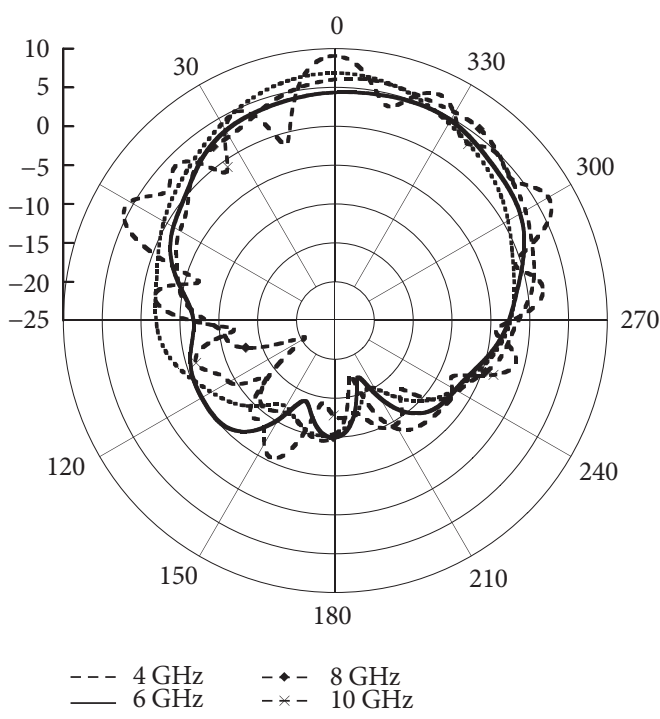

(b)

FIGURE 3: Antenna radiation pattern, on the $x-z$ horizontal plane (a) and on the $y$ - $z$ vertical plane (b) at $4,6,8$, and $10 \mathrm{GHz}$.

approximation because some organs as brain and muscles utilize more blood than fat and bones.

On the basis of these assumptions, the blood increase in the lungs and in all the other body tissues, between the end diastole and end systole, is about $20 \%$ and $2 \%$, respectively. Finally, the tissues electrical parameters at the end of systole $\left(\varepsilon_{s}\right.$ and $\left.\sigma_{s}\right)$ have been calculated as an average between the value of the tissues electrical parameters at the end diastole $\left(\varepsilon_{d}\right.$ and $\left.\sigma_{d}\right)$ and the value of the blood $\left(\varepsilon_{b}=53.95\right.$ and $\left.\sigma_{b}=5.39 \mathrm{~S} / \mathrm{m}\right)$ weighted by the above reported percentages. The results are shown in Table 2.

In order to test the previously cited hypothesis on the radar operating principle a time domain simulation with the twin antenna in front of a model of the thorax has been performed (see Figure 4). In particular, the human body has been modeled by using a multilayer structure approximating the thorax of the visible human [19]. The considered geometry has been simulated by means of the CAD Microwave Studio by CST. The antenna was excited at one port by a Gaussian pulse with a $3-10 \mathrm{GHz}$ bandwidth and the signal time behavior was recorded at the other port.

The comparison between the recorded signals, achieved by assigning to the various tissues the parameter values at the end diastole end at the end systole, shows signal differences of few microvolts, not detectable by a UWB radar.

Concerning the micromovements of the skin, according to [15], a displacement between the antenna and the skin of $\pm 30 \mu \mathrm{m}$ has been supposed and the structure has been simulated by means of Microwave Studio. In particular a reference situation with the antenna placed at $0.5 \mathrm{~mm}$ far from the body has been considered. Then the distance between the antenna and the body has been varied in the $\pm 30 \mu \mathrm{m}$ range. The signal received by the antenna at port 2 ,
TABLE 2: Tissues electrical parameters at the end of diastole $\left(\varepsilon_{d}\right.$ and $\left.\sigma_{d}\right)$ and at the end of systole $\left(\varepsilon_{s}\right.$ and $\left.\sigma_{s}\right)$.

\begin{tabular}{lcccc}
\hline & $\varepsilon_{d}$ & $\varepsilon_{s}$ & $\sigma_{d}(\mathrm{~S} / \mathrm{m})$ & $\sigma_{s}(\mathrm{~S} / \mathrm{m})$ \\
\hline Skin & 35.77 & 36.13 & 3.06 & 3.11 \\
Muscle & 49.54 & 49.63 & 4.04 & 4.07 \\
Heart wall & 50.27 & 50.34 & 4.86 & 4.87 \\
Lung & 44.86 & 46.68 & 3.94 & 4.23 \\
\hline
\end{tabular}

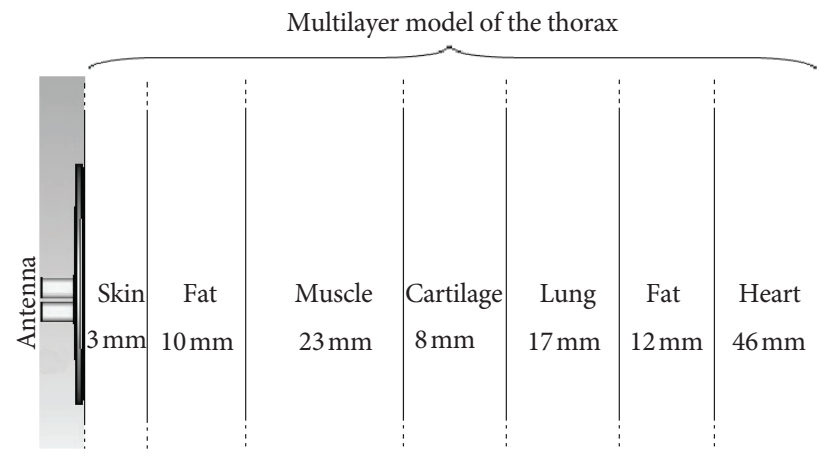

FIGURE 4: Twin antenna in front of a multilayer model of the thorax.

exciting at port 1 as in the previous study, is depicted in Figure 5 . The figure shows that up to $1.5 \mathrm{~ns}$ all the signals are perfectly superimposed (the signal is inside the antenna) and then they split. The body micromovements are converted in delays that give rise to amplitude differences up to $20 \mathrm{mV}$ (see Figure 5, absolute difference). In particular there is a direct correlation between the delays and the body micromovements.

It is worth noting that this effect is not directly connected to the spatial displacement, as in the classical radar analysis, but it is due to a modification of the complex antenna 


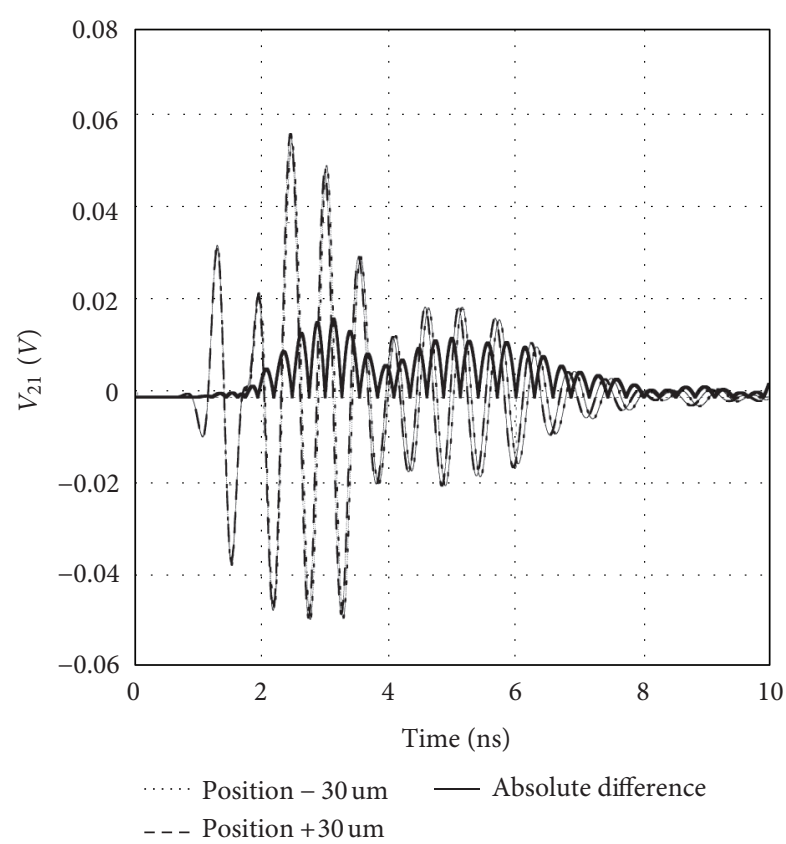

Figure 5: $V_{21}$ simulated for different displacement between the thorax and the antenna.

radiation impedance. Moreover, it is interesting to note that the absolute difference signal can be recovered in more than one time point between 2 and $8 \mathrm{~ns}$.

\section{Discussion}

The performed study has evidenced the ability of the twin antenna to detect heart activity by exiting the twin antenna at one port and measuring the reflections at the other port. A question arises: is this antenna operation better than the one in which the antenna transmits and receives with the same arms?

This issue has been studied by placing the antenna in front of the multilayer body model (see Figure 4), exciting the antenna with a Gaussian pulse with a $3-10 \mathrm{GHz}$ bandwidth and measuring the signal at the transmitting arms $\left(V_{11}\right)$ before and after a distance variation of $30 \mu \mathrm{m}$. The obtained results are reported in Figure 6 together with the absolute differences between the two signals.

The maximum voltage difference on the $V_{11}$ signals is about $40 \mathrm{mV}$ as compared with the $20 \mathrm{mV}$ on $V_{21}$ (see Figure 5 ), but the percentage difference, normalized respect to the maximum of the whole signal, is $16 \%$ for $V_{11}$, and $30 \%$ for $V_{21}$. Hence the signal variation $V_{21}$ can be better evaluated than $V_{11}$ by the sampling circuit used in the UWB radar.

In conclusion, the use of two cross-polarized radiating structures for the transmission and the reception of the UWB signals makes the proposed antenna better than a similar one using a single radiator. Moreover, with respect to similar realizations using two bow-tie, horn, or Vivaldi radiators [3] the proposed structure is very compact with a beam propagation axis perpendicular to the substrate. This makes the twin antenna easy to integrate in the UWB radar

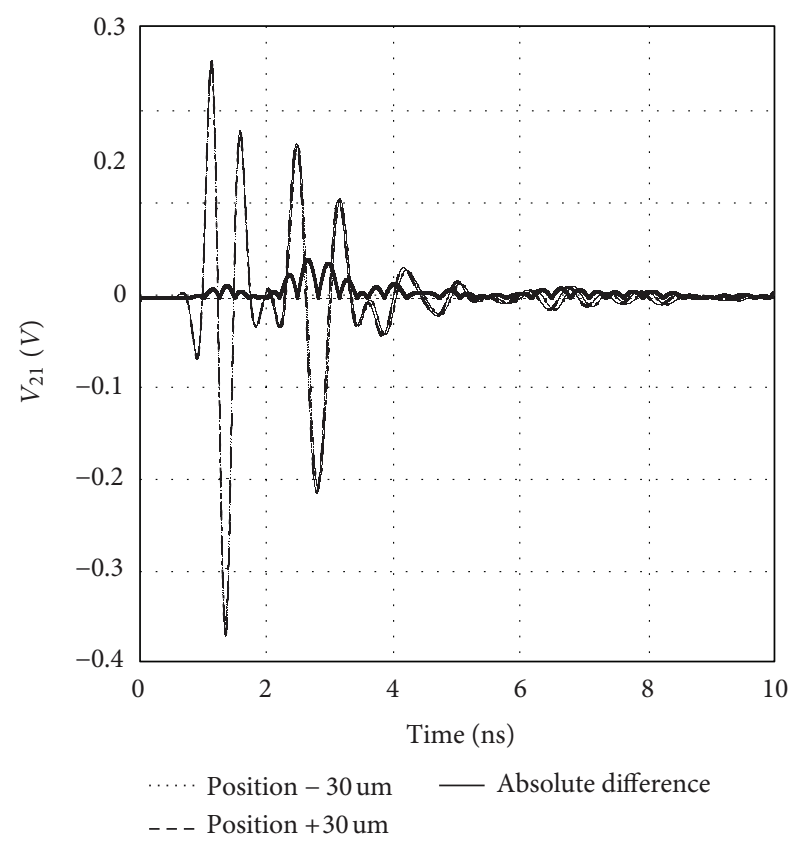

Figure 6: $V_{11}$ simulated for different displacement between the thorax and the antenna.

electronics and it makes easier the realization of a wearable radar.

\section{Conclusions}

The proposed twin antenna is a variation of the famous Archimedean spiral antenna, with two arms used for transmission and other two arms used for capturing the received echo.

The reflection coefficient at the feed point of the antenna is lower than $-8 \mathrm{~dB}$ over the $3-12 \mathrm{GHz}$ band while the twoantennas coupling is about $-20 \mathrm{~dB}$.

If inserted in a range gating UWB radar the antenna can be used to detect the heart rate activity.

In a UWB radar for heart rate activity monitoring, the measured signal is not related to an echo coming from the heart wall, but seems to be related to a change in the antenna radiating impedance as a consequence of the microdisplacements of the thorax in front of the antenna produced by the heart movements.

\section{References}

[1] T. E. McEwan, "Ultra-wideband radar motion sensor," United States Patent 5,361,070, 1994.

[2] E. M. Staderini, “UWB radars in medicine," IEEE Aerospace and Electronic Systems Magazine, vol. 17, no. 1, pp. 13-18, 2002.

[3] I. Y. Immoreev, S. Samkov, and T. H. Tao, "Short-distance ultrawideband radars," IEEE Aerospace and Electronic Systems Magazine, vol. 20, no. 6, pp. 9-14, 2005.

[4] S. Allen, J. C. Lin, J. B. Anderson et al., "ICNIRP statement on EMF-emitting new technologies," Health Physics, vol. 94, no. 4, pp. 376-392, 2008. 
[5] S. Pisa, P. Bernardi, M. Cavagnaro, E. Pittella, and E. Piuzzi, "A circuit model of an ultra wideband impulse radar system for breath-activity monitoring," International Journal of Numerical Modelling, vol. 25, no. 1, pp. 46-63, 2012.

[6] P. J. Gibson, "The Vivaldi aerial," in Proceedings of the 9th European Microwave Conference, pp. 101-105, Brighton, UK, September 1979.

[7] E. Guillanton, J. Y. Dauvignac, C. Pichot, and J. Cashman, "A new design tapered slot antenna for ultra-wideband applications," Microwave and Optical Technology Letters, vol. 19, no. 4, pp. 286-289, 1998.

[8] E. Pittella, P. Bernardi, M. Cavagnaro, S. Pisa, and E. Piuzzi, "Design of UWB antennas to monitor cardiac activity," Journal of the Applied Computational Electromagnetics Society, vol. 26, no. 4, pp. 267-274, 2011.

[9] G. Cappelletti, D. Caratelli, R. Cicchetti, and M. Simeoni, "A low-profile printed drop-shaped dipole antenna for wideband wireless applications," IEEE Transactions on Antennas and Propagation, vol. 59, no. 10, pp. 3526-3535, 2011.

[10] S. Jacobsen, H. O. Rolfsnes, and P. R. Stauffer, "Characteristics of microstrip muscle-loaded single-arm Archimedean spiral antennas as investigated by FDTD numerical computations," IEEE Transactions on Biomedical Engineering, vol. 52, no. 2, pp. 321-330, 2005.

[11] L. Stutzman and G. A. Thiele, Antenna Theory and Design, Wiley, New York, NY, USA, 2nd edition, 1998.

[12] X. Yun, E. C. Fear, and R. H. Johnston, "Compact antenna for radar-based breast cancer detection," IEEE Transactions on Antennas and Propagation, vol. 53, no. 8, pp. 2374-2380, 2005.

[13] X. Yun, R. H. Johnston, and E. C. Fear, "Radar-based microwave Imaging for breast cancer detection: tumor sensing with crosspolarized reflections," in Proceedings of the IEEE Antennas and Propagation Society Symposium 2004 Digest held in Conjunction with: USNC/URSI National Radio Science Meeting, vol. 3, pp. 2432-2435, June 2004.

[14] T. E. McEwan, "Body monitoring and imaging apparatus and method," United States Patent 5,573,012.

[15] E. M. Staderini and G. Varotto, "On the UWB medical radars working principle," International Journal of Ultra Wideband Communications and Systems, vol. 2, no. 2, pp. 83-93, 2011.

[16] First Report and Order: Revision of Part 15 of the Commission's Rules Regarding Ultra-Wideband Transmission Systems Federal Communications Commission (FCC), ET Docket 98153, 2002.

[17] H. A. Hjortland, D. T. Wisland, T. S. Lande, C. Limbodal, and K. Meisal, "CMOS impulse radar," in Proceedings of the 24th Norchip Conference, pp. 75-79, November 2006.

[18] D. Lamensdorf and L. Susma, "Baseband-pulseantenna techniques," IEEE Antennas and Propagation Magazine, vol. 36, no. 1, pp. 20-30, 1994.

[19] Visible Human Project, http://www.nlm.nih.gov/research/ visible/visible_gallery.html, 2012. 

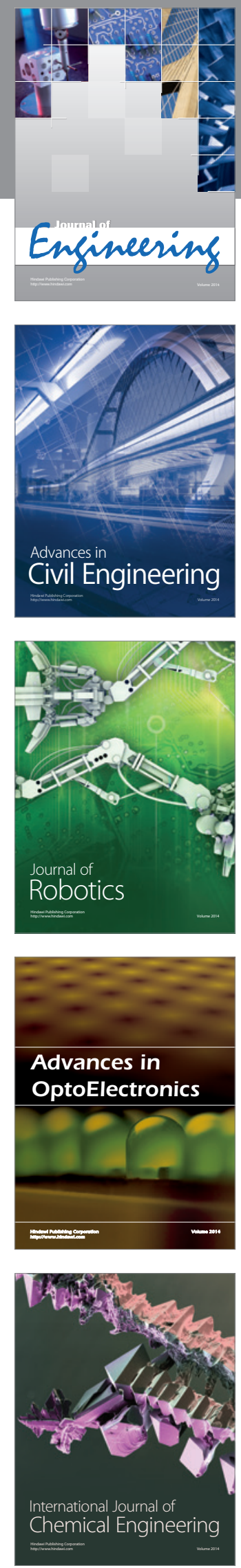

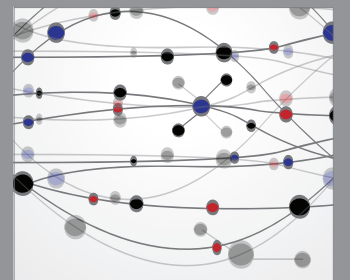

The Scientific World Journal
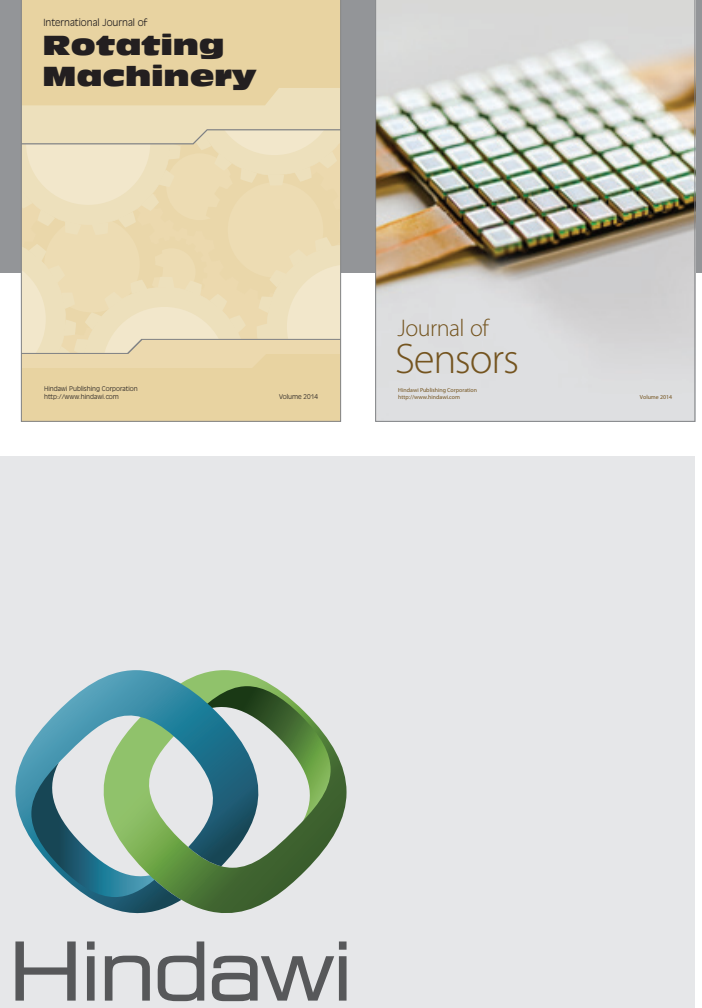

Submit your manuscripts at http://www.hindawi.com
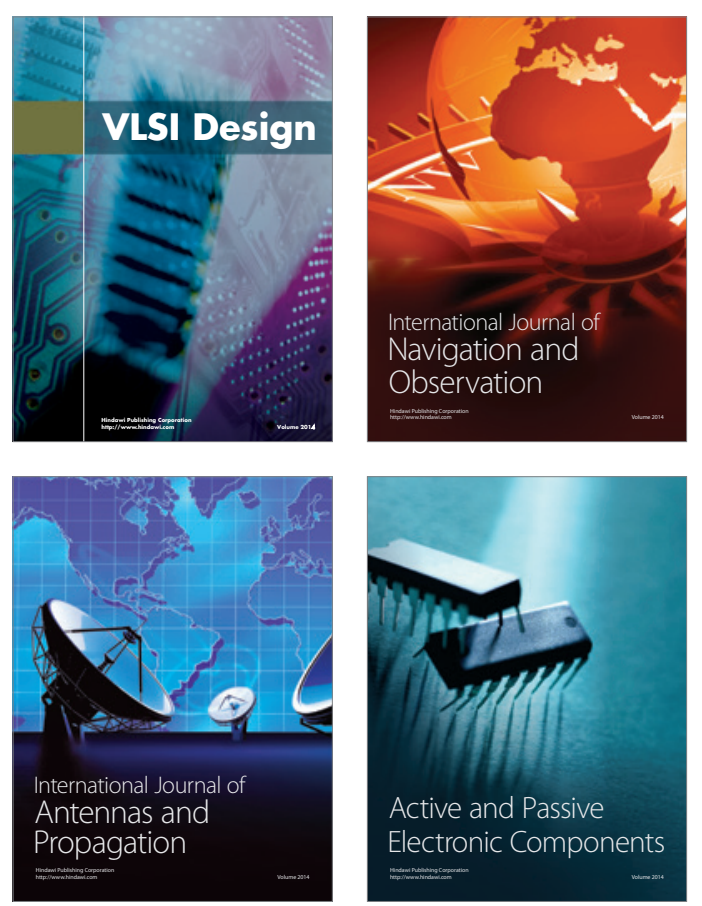
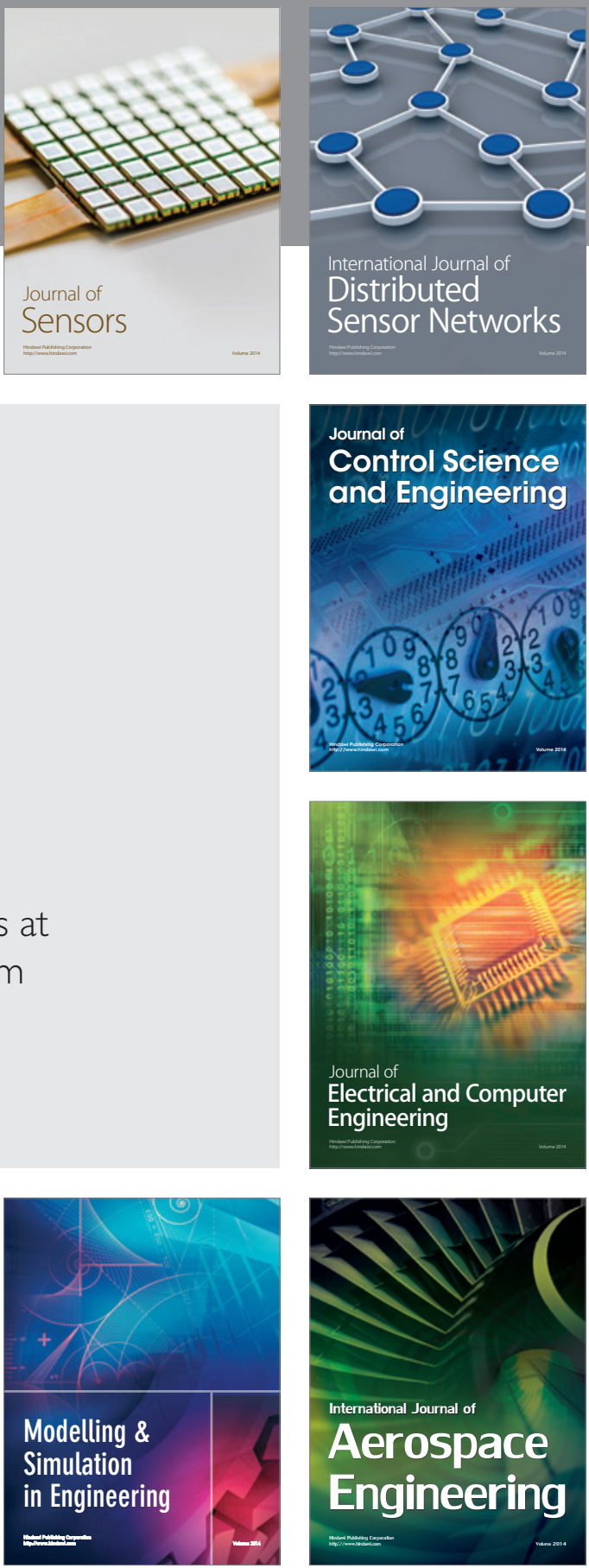

Journal of

Control Science

and Engineering
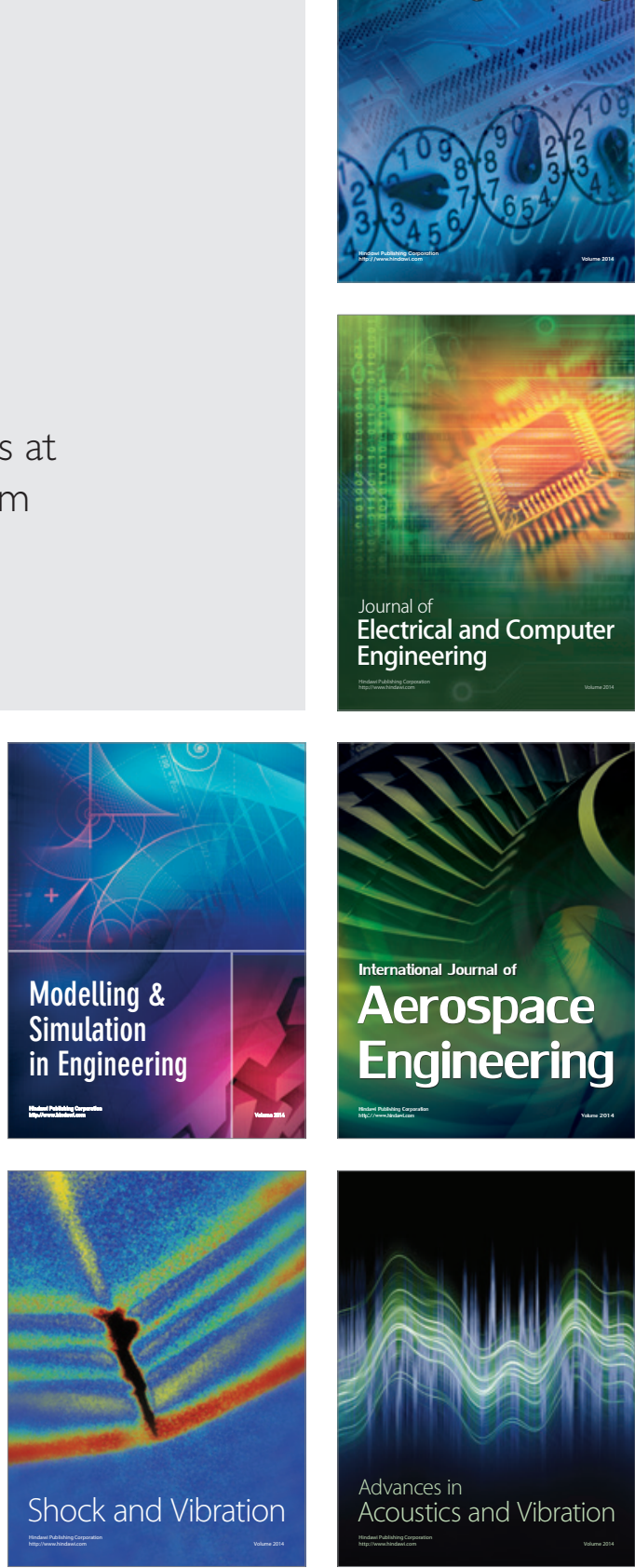